\title{
The methods for processing and storage of data in global navigation satellite systems for the task of perimeter protection
}

\author{
Alexey Samoylov ${ }^{1,}$, Andrey Kostyuk ${ }^{1}$, Viktor Potapov ${ }^{1}$, and Yuriy Borodyansky ${ }^{2}$ \\ ${ }^{1}$ Southern Federal University, Rostov-on-Don, Russia \\ ${ }^{2}$ The Bonch-Bruevich Saint-Petersburg State University of Telecommunications, Saint-Peterburg, \\ Russia
}

\begin{abstract}
The methods for processing and storage of GNSS data for the task of perimeter protection are described. The article proposes a basic algorithm for the operation of a GNSS data collection, processing and storage system that allows you to track processed GNSS data on a remote web service in public formats, as well as send signals through a client mobile application to available platforms and systems about temporarily suspending or canceling GNSS data recording.
\end{abstract}

\section{Introduction}

For the purpose of protecting the perimeter of a production facility of large territorial extent, taking into account the possible presence of threats of unauthorized physical penetration, on the territory of which stationary or mobile objects are located, the use of uninhabited intelligent interacting mobile robotic platforms is required. To ensure the protection of an extended perimeter, it is necessary to solve a number of tasks, including:

- creation of a database of the perimeter security system (DB PSS) for storing and updating information about the objects of the security system and threats from potential offenders (PO);

- development of a subsystem for modeling a perimeter security system (SMPSS) for planning joint operation of protected area objects;

- development of means of information exchange between the database and SMPSS;

- integration into one of the modern geographic information systems (GIS).

In the previously published works [1 - 10] the structure and purpose of the DB PSS are described and the use of graph approaches for the implementation of the modeling subsystem is proposed. The purpose of the study in this article is to study the principles of creating a system for collecting, processing and storing GNSS (global navigation satellite systems) data for the tasks of protecting the perimeter of mobile or other robotic platforms and systems in real time.

Along with the development of GNSS equipment, there is also progress in the development of microcontrollers. Thanks to the emergence of new types of microcontrollers,

\footnotetext{
*Corresponding author: asamoylov@sfedu.ru
} 
it became possible to combine a large number of tasks, including receiving and sending data to many devices at the same time, automating work both on a local and wireless network, up to monitoring the security of the perimeter of robotic systems and platforms of an entire enterprise. However, managing a large security company is often impossible without web technologies, in particular, services for managing large data streams. Web technologies have progressed steadily, as often the variety of virtual and dedicated computing power has made it possible to operate a large security company in different parts of the world.

One of the examples of the application of a set of GNSS equipment, microcontrollers and web-technologies, in particular, will be a system for collecting, processing and storing GNSS data for perimeter security tasks (SPSD). SPSD is a new innovative solution that replaces the usual human way of protecting an enterprise with an automated complex for monitoring the perimeter security of robotic systems and platforms anywhere in the world, which reduces the human factor to a minimum.

The relevance of the SPSD is to control this system anywhere in the world from your personal computer, smartphone or other device that supports web technologies and the Internet, in particular. Microcontrollers and web-technologies remote virtual and dedicated servers, the user will have access to a unit for monitoring and managing GNSS data streams from all robotic systems and platforms that include in the protected area.

The purpose of the system for collecting, processing and storing GNSS data is to collect GNSS data using a standard microcontroller wirelessly from mobile or other robotic platforms and systems for further sending and post-processing of data to a remote web service via an Internet connection. This also includes database management [1] with a web-client software part or a web-interface of the server part through Internet technologies.

\section{Description of the observed object}

The considered object of observation is a protected area with mobile and other robotic platforms and systems, a block of elements of a protected area, a control and distribution unit for GNSS data from mobile or other robotic platforms and systems, as well as a web service for processing GNSS data and a mobile or other device with support web technologies.

The facility includes:

- protected area with mobile and other robotic platforms and systems [2];

- block of elements of the protected area;

- block for control and distribution of GNSS data from mobile or other robotic platforms and systems;

- web service for processing GNSS data;

- mobile or other web-enabled device.

Each mobile or other robotic platform and system consists of:

- GNSS receiver;

- transceiver.

The block of elements of the protected area includes: a workshop with robotic production, cars, a helicopter, drones, patrol robotic platforms and other security systems with built-in or easily mounted GNSS equipment. The block for control and distribution of GNSS data from mobile or other robotic platforms and systems includes: a control microcontroller, a receiving-transmitting device (transceiver) for transmitting control signals to a block of elements of a protected area, receiving GNSS data from a block of elements of a protected area, as well as for sending the received GNSS data to a virtual or dedicated web service.

The web service includes: a virtual or dedicated web server, a GNSS data database, and a GNSS data processor. Each mobile or other robotic platform or system is equipped with a GNSS receiver to record GNSS data. Communication and data exchange of the elements of 
the protected area with the control microcontroller is carried out both wirelessly and by wire. Raw GNSS data coming from all elements of the protected area are distributed in the control microcontroller into groups and sent to a web service via an Internet connection and entered into a database. A client application is installed on the user's device, through which the SPSD is monitored and managed. The GNSS data processing web service performs the following functions: receiving GNSS data from the elements of the protected area through the control microcontroller, entering the received data into the SQL database, as well as sending control signals to stop or enable recording of certain types of elements of the protected area.

The user has access to a web interface, namely: a database and processed GNSS data, displaying objects on the map in real time using auxiliary software, editing data. Data editing in this case means loading and deleting GNSS and processed data for the selected element type. In addition, the user will have access to the control unit for the main microcontroller, namely: turning on and off the recording of GNSS data for the selected elements. Each mobile or other robotic platform and system performs the following functions: transmitting GNSS data to the control microcontroller via a wireless or wired network.

\section{The structure of the information object}

The SPSD information object contains:

- control signals for each mobile or other robotic platform and system to control GNSS data streams;

- control signals for the block of elements of the protected area to control signals to stop or enable recording of GNSS data;

- control signals for the control and distribution unit of GNSS data from mobile or other robotic platforms and systems to control the reception of GNSS data;

- control signals for the control and distribution unit of GNSS data from mobile or other robotic platforms and systems to control the flow of GNSS data from the elements of the protected area;

- control signals for the control unit and distribution of GNSS data from mobile or other robotic platforms and systems to control the sending of GNSS data;

- control signals for a web service to control GNSS data processing;

- control signals for the web service for managing the SQL database;

- control signals for a mobile or other device with support for web technologies to control a web service via the Internet connection through browsers;

- control signals for a mobile or other device with support for web technologies to control a web service through a client application.

\section{Target function of the system}

The target function of the system for collecting, processing and storing GNSS data for perimeter security tasks is the collection of GNSS data from the elements of the protected area, the management of GNSS data flows and distribution by types of elements of the protected area, sending GNSS data to a cloud web service for post-processing and entering data into SQL-database. GNSS data streams are controlled wirelessly or wired from a mobile or other device that supports web technology or a client application. Control means access to a web service, namely: web interface, SQL database, enabling / disabling GNSS data recording on the elements of the protected area in real time. Functions performed by SPSD:

- reading GNSS data from GNSS equipment; 
- reading an electrical signal, namely turning on / off the recording of GNSS data supplied by the user from a mobile or other device that supports web technologies or a client application.

- formation of GNSS data packets;

- exchange of GNSS packets of data from the elements of the protected area via wireless or wired communication with a control microcontroller;

- data exchange of the controlling microcontroller with a web service via wireless or wired communication;

- processing of GNSS data by a web service;

- post-processing of GNSS data streams into a common navigation format;

- flow control (loading / deleting) of GNSS data from the elements of the protected area through a web service or client application via wireless or wired communication;

- visualization of post-processed GNSS data on the map.

At each element of the protected area, the installed GNSS equipment reads raw GNSS data and transmits it through the transceiver to the control microcontroller. The control microcontroller groups the collected raw GNSS data into types and transmits it to a web service via an Internet connection, where post-processing and data entry into the SQL database takes place. On the web service, the user can control the streams of processed GNSS data, view the contents of the SQL database, namely the post-processed GNSS data on the map, and also send electrical signals to stop or turn on the necessary elements of the protected area by sending an electrical signal to the control microcontroller. The electrical signal is read out on the control microcontroller, where the desired GNSS data stream is turned off/on. Through wireless and wired communication, both the reception and transmission of GNSS data streams and two-channel sending of electrical signals to control the GNSS data streams take place. Post-processing of GNSS data is carried out using three methods, namely: using available post-processing web services, open software implementation and research software implementation.

\section{Generalized algorithm for the functioning of the SDOK system for the task of perimeter protection}

The presented SPSD system has the following operating modes:

- "Standby mode" operating mode when there is no connection with the web service and elements of the protected area for more than 10 minutes. In this mode, repeated attempts are made to connect to the web service and elements of the protected area, after 10 minutes of unsuccessful attempts, an SMS notification is sent to the user and the periphery is terminated before the user makes a decision.

- "Notification mode" operating mode when the user is absent for more than 30 minutes online. In this mode, the session time with the user is counted and after 30 minutes an SMS notification is sent to the user. This mode also notifies the user if the microcontroller's connection with the web service fails.

- "RTK mode" operating mode when the user is on the web service or client application. This mode of operation is activated automatically when the user is online on the web service or client application of the SPSD system.

The block diagram of the generalized algorithm for the functioning of the SPSD system can be presented as follows (fig. 1).

1) Initialization of the SPSD system begins with powering up the main microcontroller and loading the firmware, then the transceiver, coupled with the microcontroller, creates an access point, and is also identified on the Internet. 
2) The prepared SPSD system establishes communication with the web server and the elements of the protected area.

3) The SPSD system determines the consistency of the connection with the web server and elements of the protected area.

4) If the agreement did not occur with the web server and the elements of the protected area, then the SPSD system goes into standby mode.

5) There are repeated approvals by the SPSD system with the web-server and the elements of the protected area within 10 minutes.

6) After 10 minutes of unsuccessful coordination with the web server and the elements of the protected area, the SPSD system shuts down the operation of the periphery and sends an SMS notification to the user.

7) The prepared SPSD system, having established a connection with the web server and elements of the protected area, allows the user to manually connect to the wireless microcontroller module via a web service or a mobile application.

8) The SPSD system determines the consistency of the connection with the user or his presence on the web server.

9) If the definition of the user is unsuccessful, then the SPSD system goes into notification mode.

10) There are repeated definitions of the user by the SPSD system within 30 minutes.

11) After 30 minutes of unsuccessful user identification, the SPSD system sends an SMS notification to the user.

12) The prepared SPSD system, having established a connection with the user, switches to the real-time kinematics mode.

13) The SPSD system determines whether a command signal is received to control the elements of the protected area.

14) The block of elements of the protected area is responsible for receiving GNSS data from the elements of the protected area, as well as receiving command signals from the microcontroller.

15) The GNSS data control and distribution unit is responsible for receiving command signals from the user from the web server or mobile application to control GNSS equipment on the elements of the protected area. The block also distributes the received raw GNSS data and states of GNSS equipment by types and sends data and states to the web service, as well as the states are available in the mobile application.

16) The SPSD system determines whether a command signal has come to the control unit, when a command signal is received, the elements of the protected area are sent to the unit.

17) Received raw GNSS data by web server is processed by web server GNSS data. 


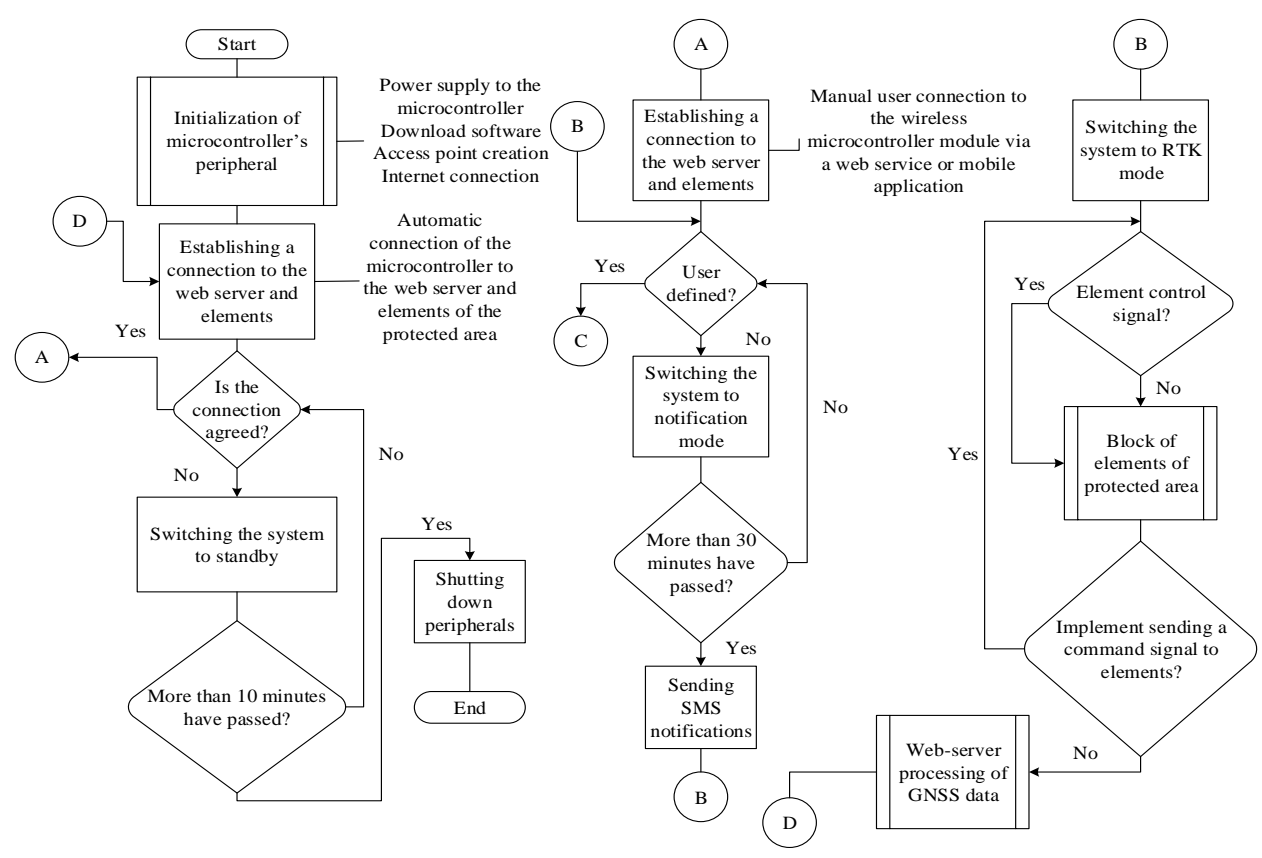

Fig. 1. Generalized algorithm for the functioning of the system.

\section{Algorithm for web processing of GNSS data}

The block diagram of the web-processing algorithm of the GNSS system is shown in figure

2. Let's analyze this algorithm in more depth in separate blocks:

1) Web server receives raw GNSS data from the main microcontroller;

2) Raw GNSS data is post-processed using web services;

3) Raw GNSS data is post-processed using open source software;

4) Raw GNSS data is post-processed using research software implementation;

5) The web server determines the readiness of processing;

6) In the absence of a processed package, the web server waits for GNSS data processing from other methods;

7) Processed and raw GNSS data is sent to the data warehouse;

8) The web server is used to enter information about the processed and initial data into the SQL database;

9) In parallel, the web server receives information from the main microcontroller about the operability of the GNSS equipment;

10) The received information about the operability of the GNSS equipment is entered and displayed in the web-control panel;

11) The user can change the states of GNSS equipment and send signals-commands through the web-based control panel. 


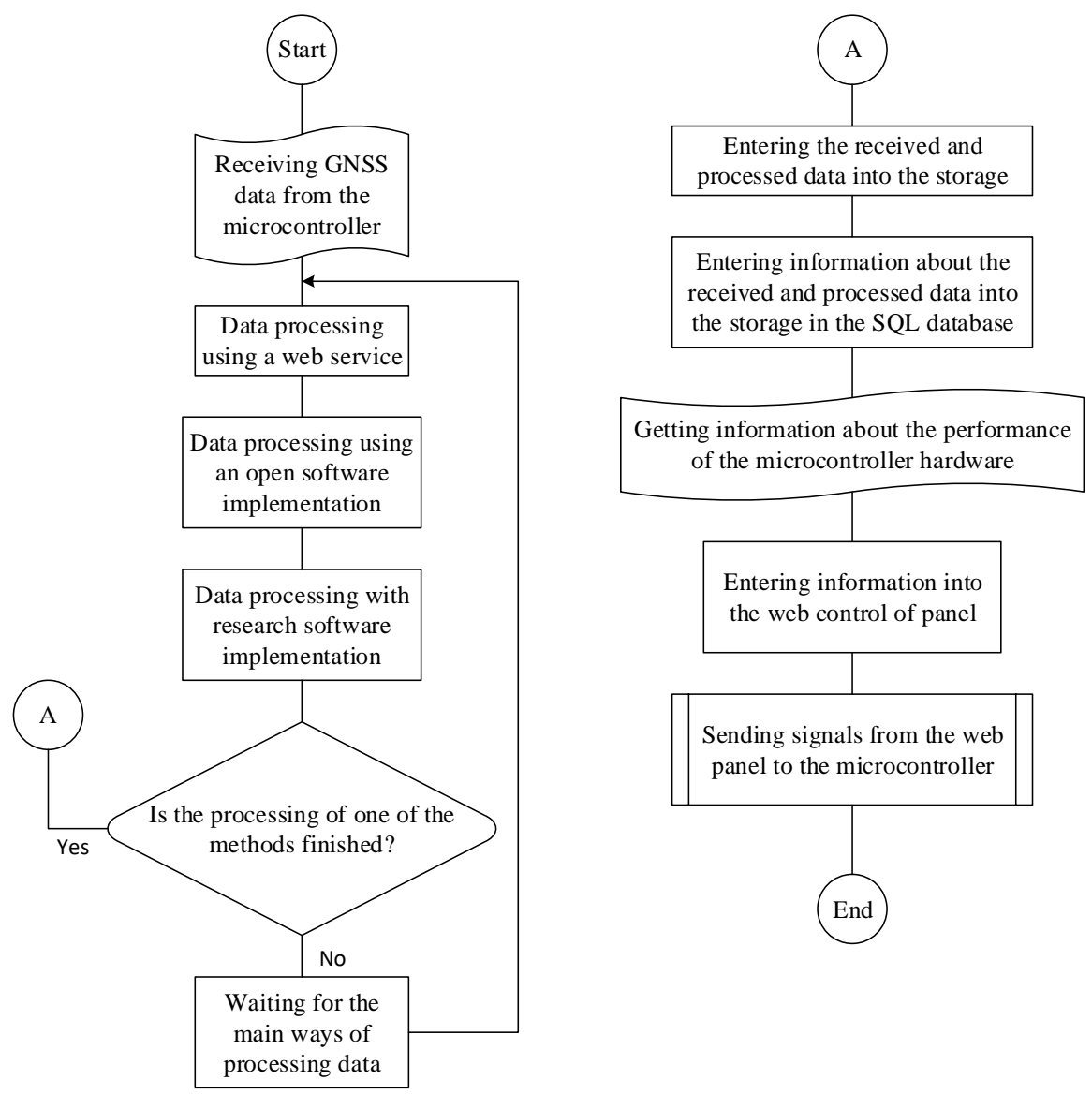

Fig. 2. Algorithm of GNSS data web processing.

Thus, the proposed principle of constructing a system for processing and storing GNSS data for the task of perimeter security allows users from a mobile or other device that supports web technologies and the Internet to track processed GNSS data on a remote web service in public formats, as well as send signals via client mobile application available by platforms and systems on temporary suspension or cancellation of GNSS data recording.

\section{Acknowledgments}

The reported study was funded by RFBR according to the research project № 20-07-00559.

\section{References}

1. Kostyuk A I 2017 Modern high technology 1243 - 48

2. Kostyuk A I, Shapoval N E 2017 Collection of articles of the II All-Russian scientificpractical conference of young scientists, graduate students, undergraduates and students "Information systems and technologies: fundamental and applied research" (Taganrog) p 448

3. Kostyuk A I 2017 Modern high technology 658 - 61 ISSN: 1812 - 7320 http://wwwtoptechnologiesru/ru/article/ view?id=36698 
4. Lebedev B K, Lebedev O B, Lebedeva E M, Kostyuk A I 2019 Artificial Intelligence and Algorithms in Intelligent Systems CSOC2018 2018 Advances in Intelligent Systems and Computing $764 \mathrm{https}$ ://wwwscopuscom/authid/detailuri?authorId=57196048780

5. Kostyuk A I, Muntyan E R, Polenov M Yu 2015 News SFU Technical science 346 54 ISSN: $1999-9429$

6. Kostyuk A I, Polenov M Yu, Muntyan E R, Lukyanov V A, Nikolava A Yu 2015 Informatization and communication 372 - 77 ISSN: 2078 - 8320

7. Lebedev B K, Lebedev O B, Lebedeva E O, Kostyuk A I Proceedings of the Second International Scientific Conference "Intelligent Information Technologies for Industry” (IITI'17) IITI 2017 Advances in Intelligent Systems and Computing 679388 - 398 https://linkspringercom/chapter/101007/978-3-319-68321-8_40

8. Polenov M Yu, Kostyuk A I, Lukyanov V A 2015 Proceedings of the XII All-Russian Scientific Conference (Rostov-on-Don: Publishing House of SFU) $176-78$

9. Muntyan E R, Kostyuk A I, Liotweisen V V 2015 The innovative development of modern science: a collection of articles of the International scientific-practical conference (Ufa: Aeterna) p 49

10. Kostyuk A I, Bespalov D A, Romanov V V 2020 Technical Sciences 2(261) 74 - 79 